\title{
The Perturbation Bound for the Spectral Radius of a Nonnegative Tensor
}

\author{
Wen $\mathrm{Li}^{1}$ and Michael $\mathrm{K} . \mathrm{Ng}^{2}$ \\ ${ }^{1}$ School of Mathematical Sciences, South China Normal University, Guangzhou, China \\ ${ }^{2}$ Department of Mathematics, Hong Kong Baptist University, Hong Kong
}

Correspondence should be addressed to Michael K. Ng; mng@math.hkbu.edu.hk

Received 29 March 2014; Revised 29 August 2014; Accepted 9 September 2014; Published 28 September 2014

Academic Editor: Nils Henrik Risebro

Copyright (c) 2014 W. Li and M. K. Ng. This is an open access article distributed under the Creative Commons Attribution License, which permits unrestricted use, distribution, and reproduction in any medium, provided the original work is properly cited.

\begin{abstract}
We study the perturbation bound for the spectral radius of an $m$ th-order $n$-dimensional nonnegative tensor $\mathscr{A}$. The main contribution of this paper is to show that when $\mathscr{A}$ is perturbed to a nonnegative tensor $\widetilde{A}$ by $\Delta \mathscr{A}$, the absolute difference between the spectral radii of $\mathscr{A}$ and $\widetilde{A}$ is bounded by the largest magnitude of the ratio of the ith component of $\Delta \mathscr{A} \mathbf{x}^{m-1}$ and the ith component $\mathbf{x}^{m-1}$, where $\mathbf{x}$ is an eigenvector associated with the largest eigenvalue of $\mathscr{A}$ in magnitude and its entries are positive. We further derive the bound in terms of the entries of $\mathscr{A}$ only when $\mathbf{x}$ is not known in advance. Based on the perturbation analysis, we make use of the NQZ algorithm to estimate the spectral radius of a nonnegative tensor in general. On the other hand, we study the backward error matrix $\Delta \mathscr{A}$ and obtain its smallest error bound for its perturbed largest eigenvalue and associated eigenvector of an irreducible nonnegative tensor. Based on the backward error analysis, we can estimate the stability of computation of the largest eigenvalue of an irreducible nonnegative tensor by the NQZ algorithm. Numerical examples are presented to illustrate the theoretical results of our perturbation analysis.
\end{abstract}

\section{Introduction}

Let $\mathbb{R}$ be the real field. We consider an $m$ th order $n$ dimensional tensor $\mathscr{A}$ consisting of $n^{m}$ entries in $\mathbb{R}$ :

$$
\mathscr{A}=\left(a_{i_{1}, i_{2}, \ldots, i_{m}}\right), \quad a_{i_{1}, i_{2}, \ldots, i_{m}} \in \mathbb{R}, 1 \leq i_{1}, i_{2}, \ldots, i_{m} \leq n .
$$

$\mathscr{A}$ is called nonnegative (or, resp., positive) if $a_{i_{1}, i_{2}, \ldots, i_{m}} \geq 0$ (or, resp., $\left.a_{i_{1}, i_{2}, \ldots, i_{m}}>0\right)$. In the following discussion, for a given vector $\mathbf{y}=\left(y_{1}, y_{2}, \ldots, y_{n}\right)^{T} \in \mathbb{R}^{n}$, we define a tensor-vector multiplication $\mathscr{A} \mathbf{y}^{m-1}$ to be $n$-dimensional column vector with its $i_{1}$ th entries given by

$$
\sum_{i_{2}, \ldots, i_{m}=1}^{n} a_{i_{1}, i_{2}, \ldots, i_{m}} y_{i_{2}} \cdots y_{i_{m}}, \quad 1 \leq i_{1} \leq n
$$

Also we let $\mathbb{P}=\left\{\left(y_{1}, y_{2}, \ldots, y_{n}\right) \mid y_{i} \geq 0\right\}$ be the positive cone, and we let the interior of $\mathbb{P}$ be denoted as $\operatorname{int}(\mathbb{P})=\left\{\left(y_{1}, y_{2}, \ldots, y_{n}\right) \mid y_{i}>0\right\}$. When $\mathbf{y} \in \mathbb{P}$ (or $\mathbf{y} \in \operatorname{int}(\mathbb{P})), \mathbf{y}$ is a nonnegative (or a positive) vector. We denote the $i$ th component of $\mathbf{y}$ by $(\mathbf{y})_{i}$ or $y_{i}$, and we denote the $\left(i_{1}, i_{2}, \ldots, i_{m}\right)$ th entry of $\mathscr{A}$ by $(\mathscr{A})_{i_{1}, i_{2}, \ldots, i_{m}}$ or $a_{i_{1}, i_{2}, \ldots, i_{m}}$.

In recent studies of numerical multilinear algebra, eigenvalue problems for tensors have brought special attention. There are many related applications in information retrieval and data mining; see, for instance, $[1,2]$.

Definition 1. Let $\mathscr{A}$ be an $m$ th-order $n$-dimensional tensor and let $\mathbb{C}$ be the set of all complex numbers. Assume that $\mathscr{A} \mathbf{x}^{m-1}$ is not identical to zero. We say $(\lambda, \mathbf{x}) \in \mathbb{C} \times\left(\mathbb{C}^{n} \backslash\{0\}\right)$ is an $H$-eigenpair of $\mathscr{A}$ if

$$
\mathscr{A} \mathbf{x}^{m-1}=\lambda \mathbf{x}^{[m-1]} .
$$

Here, $\mathbf{x}^{[\alpha]}:=\left(x_{1}^{\alpha}, x_{2}^{\alpha}, \ldots, x_{n}^{\alpha}\right)^{T}$. The spectral radius $\rho(\mathscr{A})$ of $\mathscr{A}$ is defined by the largest eigenvalue of $\mathscr{A}$ in magnitude.

This definition was introduced by Qi [3] when $m$ is even and $\mathscr{A}$ is symmetric. Independently, Lim [4] gave such a definition but restricted $\mathbf{x}$ to be a real vector and $\lambda$ to be a real number. For the largest $H$-eigenvalue of a nonnegative tensor, 
the Perron-Frobenius theorem was proved by Chang et al. [5], Friedland et al. [6], and Lim [4]. Y. Yang and Q. Yang [7] generalized the weak Perron-Frobenius theorem to general nonnegative tensors. In [4], the concept of irreducibility in nonnegative matrices has been extended to nonnegative tensors.

Definition 2. An $m$ th-order $n$-dimensional tensor $\mathscr{A}$ is called reducible if there exists a nonempty proper index subset $\mathbb{\subset}$ $\{1,2, \ldots, n\}$ such that

$$
a_{i_{1}, i_{2}, \ldots, i_{m}}=0, \quad \forall i_{1} \in \mathbb{J}, \forall i_{2}, \ldots, i_{m} \notin \mathbb{J} .
$$

If $\mathscr{A}$ is not reducible, then we call $\mathscr{A}$ irreducible.

Theorem 3. Suppose $\mathscr{A}$ is an $m$ th-order $n$-dimensional nonnegative tensor.

(i) Then there exist $\lambda_{0} \geq 0$ and $\mathbf{x} \in \mathbb{P}$ such that

$$
\mathscr{A} \mathbf{x}^{m-1}=\lambda_{0} \mathbf{x}^{[m-1]}
$$

(ii) If $\mathscr{A}$ is irreducible, then $\lambda_{0}>0$ and $\mathbf{x} \in \operatorname{int}(\mathbb{P})$. Moreover, if $\lambda$ is an eigenvalue with a nonnegative eigenvector, then $\lambda=\lambda_{0}$. If $\lambda$ is an eigenvalue of $\mathscr{A}$, then $|\lambda| \leq \lambda_{0}$.

We call $\mathbf{x}$ a Perron vector of a nonnegative tensor corresponding to its largest nonnegative eigenvalue.

Some algorithms for computing the largest eigenvalue of an irreducible tensor were proposed; see, for instance, [8-10]. However, the perturbation analysis and the backward error analysis for these algorithms have not been studied, which are important to the analysis of the accuracy and stability for computing the largest eigenvalue by these algorithms.

In this paper, we are interested in studying the perturbation bound for the spectral radius of an $m$ th-order $n$ dimensional nonnegative tensor $\mathscr{A}$. The main contribution of this paper is to show that when $\mathscr{A}$ is perturbed to a nonnegative tensor $\widetilde{A}$ by $\Delta \mathscr{A}$ and $\mathscr{A}$ has a positive Perron vector $\mathbf{x}$, we have

$$
|\rho(\mathscr{A})-\rho(\widetilde{A})| \leq\left\|\Delta \mathscr{A} \times{ }_{1} D_{\mathbf{x}}^{-(m-1)} \times_{2} D_{\mathbf{x}} \times_{3} \cdots \times{ }_{m} D_{\mathbf{x}}\right\|_{\infty},
$$

where $D_{\mathbf{x}}=\operatorname{diag}\left(x_{1}, x_{2}, \ldots, x_{n}\right)$ is a diagonal matrix, for an $m$ th-order $n$-dimensional tensor $\mathscr{P}=\left(p_{i_{1}, i_{2}, \ldots, i_{m}}\right)$ and an $n$-by$n$ matrix $Q=\left(q_{i_{j}, i_{j}}\right)$, and the tensor-matrix multiplication [11] $\mathscr{R}=\mathscr{P} \times{ }_{j} Q$ is a tensor $\left(r_{i_{1}, i_{2}, \ldots, i_{m}}\right)$ of order $m$ and dimension $n$ with its entries given by

$$
r_{i_{1}, \ldots, i_{j-1}, i_{j}, i_{j+1}, \ldots, i_{m}} \equiv \sum_{i_{j}=1}^{n} p_{i_{1}, \ldots, i_{j-1}, i_{j}, i_{j+1}, \ldots, i_{m}} q_{i_{j}, i_{j}} ;
$$

the maximum norm of a tensor is defined as follows:

$$
\|\mathscr{P}\|_{\infty} \equiv \max _{1 \leq i_{i} \leq n} \sum_{i_{2}, \ldots, i_{m}=1}^{n}\left|p_{i_{1}, i_{2}, \ldots, i_{m}}\right| .
$$

The perturbation bound (6) shows that the absolute difference between the spectral radii of $\mathscr{A}$ and $\widetilde{\mathscr{A}}$ is bounded by the largest magnitude of the ratio of the $i$ th component of $\Delta \mathscr{A} \mathbf{x}^{m-1}$ and the $i$ th component $\mathbf{x}^{m-1}$. We further derive the bound based on the entries of $\mathscr{A}$ when $\mathbf{x}$ is not necessary to be known. Moreover, there is no convergence result of numerical algorithms $[9,10]$ for computing the spectral radius of a nonnegative tensor in general. We will make use of our perturbation results to estimate the spectral radius of a nonnegative tensor in general via the NQZ algorithm (see [10]).

On the other hand, we will study the backward error matrix $\Delta \mathscr{A}$ and obtain its smallest error bound of $\Delta \mathscr{A}$ for

$$
\widetilde{\mathscr{A}} \widetilde{\mathbf{x}}^{m-1}=(\mathscr{A}+\Delta \mathscr{A}) \widetilde{\mathbf{x}}^{m-1}=\widetilde{\lambda} \widetilde{\mathbf{x}}^{m-1}
$$

such that $\widetilde{\mathscr{A}}$ is an irreducible nonnegative tensor, $\tilde{\lambda}$ is the largest eigenvalue of $\widetilde{\mathscr{A}}$, and $\widetilde{\mathbf{x}}$ is a Perron vector of $\widetilde{\mathscr{A}}$ by the NQZ algorithm. Our theoretical results show that $\Delta \mathscr{A}=$ $\left(\delta a_{i_{1}, i_{2}, \ldots, i_{m}}\right)$ can be chosen as follows:

$$
\delta a_{i_{1}, i_{2}, \ldots, i_{m}}=\frac{(\mathbf{r})_{i_{1}} \cdot(\widetilde{\mathbf{x}})_{i_{2}} \cdots(\widetilde{\mathbf{x}})_{i_{m}}}{\|\widetilde{\mathbf{x}}\|_{2}^{2(m-1)}}
$$

where $\mathbf{r}=\tilde{\lambda} \widetilde{\mathbf{x}}^{[m-1]}-\mathscr{A} \widetilde{\mathbf{x}}^{m-1}$ and $\|\widetilde{\mathbf{x}}\|_{2}$ denotes the vector 2norm. By using these backward error results, we will evaluate the stability of computation of the largest eigenvalue of an irreducible nonnegative tensor by the NQZ algorithm.

The paper is organized as follows. In Section 2, we review the existing results and present the results for the perturbation bound of the spectral radius of a nonnegative tensor. In Section 3, we give the explicit expression of the backward error for the computation of the largest eigenvalue of an irreducible nonnegative tensor by the NQZ algorithm. Finally, concluding remarks are given in Section 4.

\section{The Perturbation Bound of the Spectral Radius}

Let us first state some preliminary results of a nonnegative tensor.

Lemma 4 (see [7, Lemma 5.2]). Suppose $\mathscr{A}$ is an mth-order n-dimensional nonnegative tensor. Then we have

$$
\min _{1 \leq i_{1} \leq n} \sum_{i_{2}, \ldots, i_{m}=1}^{n} a_{i_{1}, i_{2}, \ldots, i_{m}} \leq \rho(\mathscr{A}) \leq \max _{1 \leq i_{1} \leq n} \sum_{i_{2}, \ldots, i_{m}=1}^{n} a_{i_{i}, i_{2}, \ldots, i_{m}} .
$$

Lemma 5 (see [7, Lemma 5.1]). Suppose $\mathscr{A}$ is an $m$ th-order n-dimensional nonnegative tensor. If

$$
\sum_{i_{2}, \ldots, i_{m}=1}^{n} a_{i_{1}, i_{2}, \ldots, i_{m}}=c, \quad i_{1}=1, \ldots, n
$$

then $\rho(\mathscr{A})=c$. 
Lemma 6. Suppose $\mathscr{A}$ is an mth-ordern-dimensional nonnegative tensor with a positive Perron vector $\mathbf{x}$. Then one has

$$
\begin{aligned}
& \sum_{i_{2}, \ldots, i_{m}=1}^{n}\left(\mathscr{A} \times{ }_{1} D_{\mathbf{x}}^{-(m-1)} \times{ }_{2} D_{\mathbf{x}} \times{ }_{3} \cdots \times{ }_{m} D_{\mathbf{x}}\right)_{i_{1}, i_{2}, \ldots, i_{m}}=\rho(\mathscr{A}), \\
& i_{1}=1, \ldots, n \text {, }
\end{aligned}
$$

where $D_{\mathbf{x}}=\operatorname{diag}\left(x_{1}, x_{2}, \ldots, x_{n}\right)$.

Proof. By Theorem 3, we note that $\mathscr{A} \mathbf{x}^{m-1}=\lambda_{0} \mathbf{x}^{[m-1]}$ and $\lambda_{0}$ is the largest eigenvalue of $\mathscr{A}$. Therefore,

$$
\sum_{i_{2}, \ldots, i_{m}=1}^{n}\left(\mathscr{A} \times{ }_{1} D_{\mathbf{x}}^{-(m-1)} \times{ }_{2} D_{\mathbf{x}} \times{ }_{3} \cdots \times{ }_{m} D_{\mathbf{x}}\right)_{i_{1}, i_{2}, \ldots, i_{m}}=\lambda_{0} .
$$

The result follows.

Theorem 7. Suppose $\mathscr{A}$ is an mth-order n-dimensional nonnegative tensor, $\widetilde{A}=\mathscr{A}+\Delta \mathscr{A}$ is the perturbed nonnegative tensor of $\mathscr{A}$, and $\widetilde{A}$ has a positive Perron vector $\mathbf{z}$. Then one has

$$
\begin{aligned}
& \min _{1 \leq i_{i} \leq n}\left(\Delta \mathscr{A} \times{ }_{1} D_{\mathbf{z}}^{-(m-1)} \times{ }_{2} D_{\mathbf{z}} \times{ }_{3} \cdots \times{ }_{m} D_{\mathbf{z}} \mathrm{e}^{m-1}\right)_{i_{1}} \\
& \quad \leq \rho(\widetilde{A})-\rho(\mathscr{A}) \\
& \quad \leq \max _{1 \leq i_{i} \leq n}\left(\Delta \mathscr{A} \times{ }_{1} D_{\mathbf{z}}^{-(m-1)} \times{ }_{2} D_{\mathbf{z}} \times{ }_{3} \cdots \times_{m} D_{\mathbf{z}} \mathrm{e}^{m-1}\right)_{i_{1}},
\end{aligned}
$$

where $D_{\mathbf{z}}=\operatorname{diag}\left(z_{1}, z_{2}, \ldots, z_{n}\right)$ and $\mathbf{e}$ is a vector of all ones.

Proof. Let us consider the tensor $\mathscr{B}=\widetilde{\mathscr{A}}-\rho(\mathscr{A}) \mathscr{I}$, where $\mathscr{I}$ is the identity tensor.

For an $m$ th-order $n$-dimensional identity tensor $\mathscr{I}=$ $\left(i_{i_{1}, i_{2}, \ldots, i_{m}}\right)$,

$$
i_{i_{1}, i_{2}, \ldots, i_{m}}= \begin{cases}1, & i_{1}=i_{2}=\cdots=i_{m} \\ 0, & \text { otherwise. }\end{cases}
$$

It is clear that the eigenvalue of $\mathscr{B}$ is the same as the eigenvalue of $\mathscr{B} \times{ }_{1} \Sigma^{-(m-1)} \times_{2} \Sigma \times_{3} \cdots \times_{m} \Sigma$ for any positive diagonal matrix $\Sigma$. Then we obtain

$$
\begin{aligned}
\mathscr{B} & \times{ }_{1} \Sigma^{-(m-1)} \times_{2} \Sigma \times_{3} \cdots \times \times_{m} \Sigma \\
& =\widetilde{\mathscr{A}} \times{ }_{1} \Sigma^{-(m-1)} \times_{2} \Sigma \times_{3} \cdots \times \times_{m} \Sigma-\rho(\mathscr{A}) \mathscr{I} .
\end{aligned}
$$

By choosing $\Sigma=D_{\mathbf{z}}=\operatorname{diag}\left(z_{1}, z_{2}, \ldots, z_{n}\right)$, where $\mathbf{z}$ is a positive Perron vector of $\widetilde{\mathscr{A}}$, we have, for $i_{1}=1, \ldots, n$,

$$
\begin{aligned}
& \sum_{i_{2}, \ldots, i_{m}=1}^{n}\left(\mathscr{B} \times{ }_{1} D_{\mathbf{z}}^{-(m-1)} \times_{2} D_{\mathbf{z}} \times{ }_{3} \cdots \times{ }_{m} D_{\mathbf{z}}\right)_{i_{1}, i_{2}, \ldots, i_{m}} \\
& =\sum_{i_{2}, \ldots, i_{m}=1}^{n}\left(\widetilde{\mathscr{A}} \times_{1} D_{\mathbf{z}}^{-(m-1)} \times_{2} D_{\mathbf{z}} \times{ }_{3} \cdots \times{ }_{m} D_{\mathbf{z}}\right)_{i_{1}, i_{2}, \ldots, i_{m}} \\
& \quad-\rho(\mathscr{A}) \sum_{i_{2}, \ldots, i_{m}=1}^{n} \mathscr{I}_{i_{1}, i_{2}, \ldots, i_{m}} \\
& =\rho(\widetilde{\mathscr{A}})-\rho(\mathscr{A}) .
\end{aligned}
$$

On the other hand, we can deduce that, for $i_{1}=1, \ldots, n$,

$$
\begin{aligned}
& \sum_{i_{2}, \ldots, i_{m}=1}^{n}\left(\mathscr{B} \times{ }_{1} D_{\mathbf{z}}^{-(m-1)} \times{ }_{2} D_{\mathbf{z}} \times \times_{3} \cdots \times{ }_{m} D_{\mathbf{z}}\right)_{i_{1}, i_{2}, \ldots, i_{m}} \\
& =\sum_{i_{2}, \ldots, i_{m}=1}^{n}\left((\mathscr{A}+\Delta \mathscr{A}) \times{ }_{1} D_{\mathbf{z}}^{-(m-1)} \times{ }_{2} D_{\mathbf{z}} \times{ }_{3} \cdots \times{ }_{m} D_{\mathbf{z}}\right)_{i_{1}, i_{2}, \ldots, i_{m}} \\
& -\rho(\mathscr{A}) \sum_{i_{2}, \ldots, i_{m}=1}^{n} \mathscr{I}_{i_{1}, i_{2}, \ldots, i_{m}} \\
& =\sum_{i_{2}, \ldots, i_{m}=1}^{n}\left((\mathscr{A}+\Delta \mathscr{A}) \times{ }_{1} D_{\mathbf{z}}^{-(m-1)} \times{ }_{2} D_{\mathbf{z}} \times \times_{3} \cdots \times{ }_{m} D_{\mathbf{z}}\right)_{i_{1}, i_{2}, \ldots, i_{m}} \\
& -\rho(\mathscr{A}) \\
& =\sum_{i_{2}, \ldots, i_{m}=1}^{n}\left(\mathscr{A} \times{ }_{1} D_{\mathbf{z}}^{-(m-1)} \times{ }_{2} D_{\mathbf{z}} \times{ }_{3} \cdots \times{ }_{m} D_{\mathbf{z}}\right)_{i_{1}, i_{2}, \ldots, i_{m}}-\rho(\mathscr{A}) \\
& +\sum_{i_{2}, \ldots, i_{m}=1}^{n}\left(\Delta \mathscr{A} \times{ }_{1} D_{\mathbf{z}}^{-(m-1)} \times_{2} D_{\mathbf{z}} \times{ }_{3} \cdots \times \times_{m} D_{\mathbf{z}}\right)_{i_{1}, i_{2}, \ldots, i_{m}} .
\end{aligned}
$$

Taking $i_{1}=i^{*}$ such that

$$
\begin{aligned}
& \sum_{i_{2}, \ldots, i_{m}=1}^{n}\left(\mathscr{A} \times{ }_{1} D_{\mathbf{z}}^{-(m-1)} \times{ }_{2} D_{\mathbf{z}} \times{ }_{3} \cdots \times{ }_{m} D_{\mathbf{z}}\right)_{i^{*}, i_{2}, \ldots, i_{m}} \\
& \quad=\min _{1 \leq i_{1} \leq n} \sum_{i_{2}, \ldots, i_{m}=1}^{n}\left(\mathscr{A} \times{ }_{1} D_{\mathbf{z}}^{-(m-1)} \times_{2} D_{\mathbf{z}} \times_{3} \cdots \times \times_{m} D_{\mathbf{z}}\right)_{i_{1}, i_{2}, \ldots, i_{m}}
\end{aligned}
$$

and then by using (20) with $i_{1}=i^{*}$, we give the combination of (18) and (19) as follows:

$$
\begin{aligned}
& \rho(\widetilde{\mathscr{A}})-\rho(\mathscr{A}) \\
& =\sum_{i_{2}, \ldots, i_{m}=1}^{n}\left(\mathscr{A} \times{ }_{1} D_{\mathbf{z}}^{-(m-1)} \times{ }_{2} D_{\mathbf{z}} \times{ }_{3} \cdots \times{ }_{m} D_{\mathbf{z}}\right)_{i^{*}, i_{2}, \ldots, i_{m}}-\rho(\mathscr{A}) \\
& +\sum_{i_{2}, \ldots, i_{m}=1}^{n}\left(\Delta \mathscr{A} \times{ }_{1} D_{\mathbf{z}}^{-(m-1)} \times{ }_{2} D_{\mathbf{z}} \times{ }_{3} \cdots \times{ }_{m} D_{\mathbf{z}}\right)_{i^{*}, i_{2}, \ldots, i_{m}} \\
& =\min _{1 \leq i_{1} \leq n} \sum_{i_{2}, \ldots, i_{m}=1}^{n}\left(\mathscr{A} \times{ }_{1} D_{\mathbf{z}}^{-(m-1)} \times{ }_{2} D_{\mathbf{z}} \times{ }_{3} \cdots \times{ }_{m} D_{\mathbf{z}}\right)_{i_{1}, i_{2}, \ldots, i_{m}} \\
& -\rho(\mathscr{A}) \\
& +\sum_{i_{2}, \ldots, i_{m}=1}^{n}\left(\Delta \mathscr{A} \times{ }_{1} D_{\mathbf{z}}^{-(m-1)} \times{ }_{2} D_{\mathbf{z}} \times{ }_{3} \cdots \times{ }_{m} D_{\mathbf{z}}\right)_{i^{*}, i_{2}, \ldots, i_{m}} .
\end{aligned}
$$


Since the eigenvalue of $\mathscr{A} D_{\mathbf{z}}^{-(m-1)} \times_{2} D_{\mathbf{z}} \times{ }_{3} \cdots \times{ }_{m} D_{\mathbf{z}}$ is the same as the eigenvalue of $\mathscr{A}$, by using Lemma $4,(21)$ becomes

$$
\begin{aligned}
& \rho(\widetilde{\mathscr{A}})-\rho(\mathscr{A}) \\
& \leq \sum_{i_{2}, \ldots, i_{m}=1}^{n}\left(\Delta \mathscr{A} \times{ }_{1} D_{\mathbf{z}}^{-(m-1)} \times{ }_{2} D_{\mathbf{z}} \times{ }_{3} \cdots \times \times_{m} D_{\mathbf{z}}\right)_{i^{*}, i_{2}, \ldots, i_{m}} \\
& \quad \leq \max _{1 \leq i_{1} \leq n} \sum_{i_{2}, \ldots, i_{m}=1}^{n}\left(\Delta \mathscr{A} \times{ }_{1} D_{\mathbf{z}}^{-(m-1)} \times{ }_{2} D_{\mathbf{z}} \times{ }_{3} \cdots \times{ }_{m} D_{\mathbf{z}}\right)_{i_{1}, i_{2}, \ldots, i_{m}} \\
& \quad=\max _{1 \leq i_{i} \leq n}\left(\Delta \mathscr{A} \times{ }_{1} D_{\mathbf{z}}^{-(m-1)} \times{ }_{2} D_{\mathbf{z}} \times{ }_{3} \cdots \times{ }_{m} D_{\mathbf{z}} \mathrm{e}^{m-1}\right)_{i_{1}} .
\end{aligned}
$$

The right hand side of (15) is established. By using the above argument, we can show the left hand side of (15).

By Theorem 7, it is easy to obtain the following corollary.

Corollary 8. Suppose $\mathscr{A}$ is an $m$ th-order $n$-dimensional nonnegative tensor, $\widetilde{\mathscr{A}}=\mathscr{A}+\Delta \mathscr{A}$ is the perturbed nonnegative tensor of $\mathscr{A}$, and $\widetilde{\mathscr{A}}$ has a positive Perron vector $\mathbf{z}$. Then one has

$$
|\rho(\widetilde{\mathscr{A}})-\rho(\mathscr{A})| \leq\left\|\Delta \mathscr{A} \times{ }_{1} D_{\mathbf{z}}^{-(m-1)} \times{ }_{2} D_{\mathbf{z}} \times{ }_{3} \cdots \times{ }_{m} D_{\mathbf{z}}\right\|_{\infty},
$$

where $D_{\mathbf{z}}=\operatorname{diag}\left(z_{1}, z_{2}, \ldots, z_{n}\right)$.

It is noted that $\mathscr{A}$ can also be written as the perturbed tensor of $\widetilde{A}$; that is, $\mathscr{A}=\widetilde{\mathscr{A}}-\Delta \mathscr{A}$. Then, by Theorem 7 , we have the following bound.

Corollary 9. Suppose $\mathscr{A}$ is an mth-order n-dimensional nonnegative tensor with a positive Perron vector $\mathbf{x}$, and $\widetilde{\mathscr{A}}=\mathscr{A}+$ $\Delta \mathscr{A}$ is the perturbed nonnegative tensor of $\mathscr{A}$. Then one has

$$
\begin{aligned}
& \min _{1 \leq i_{i} \leq n}\left(\Delta \mathscr{A} \times{ }_{1} D_{\mathbf{x}}^{-(m-1)} \times_{2} D_{\mathbf{x}} \times{ }_{3} \cdots \times{ }_{m} D_{\mathbf{x}} \mathbf{e}^{m-1}\right)_{i_{1}} \\
& \quad \leq \rho(\widetilde{A})-\rho(\mathscr{A}) \\
& \quad \leq \max _{1 \leq i_{i} \leq n}\left(\Delta \mathscr{A} \times{ }_{1} D_{\mathbf{x}}^{-(m-1)} \times_{2} D_{\mathbf{x}} \times{ }_{3} \cdots \times{ }_{m} D_{\mathbf{x}} \mathbf{e}^{m-1}\right)_{i_{1}},
\end{aligned}
$$

where $D_{\mathbf{x}}=\operatorname{diag}\left(x_{1}, x_{2}, \ldots, x_{n}\right)$.

According to Corollary 9, we know that the absolute difference between the spectral radii of $\mathscr{A}$ and $\widetilde{A}$ is bounded by the largest magnitude of the ratio of the $i$ th component of $\Delta \mathscr{A} \mathbf{x}^{m-1}$ and the $i$ th component $\mathbf{x}^{m-1}$.

Remark 10. In the nonnegative matrix case $(m=2)$, if $A$ is irreducible nonnegative matrix with positive Perron vector $x$, then the perturbation of the eigenvalues of $\widetilde{A}=A+\Delta A$ and $A$ is given by

$$
\begin{aligned}
\min _{1 \leq i_{i} \leq n}\left(\Delta D_{x}^{-1} A D_{x} \mathbf{e}\right)_{i_{1}} & \leq \rho(\widetilde{\mathscr{A}})-\rho(\mathscr{A}) \\
& \leq \max _{1 \leq i_{i} \leq n}\left(\Delta D_{x}^{-1} A D_{x} \mathbf{e}\right)_{i_{1}} ;
\end{aligned}
$$

see [12]. It is easy to see that our perturbation result in Corollary 9 can be reduced to the bound in (25).

Remark 11. In [6], it has been shown that if $\mathscr{A}$ is symmetric and weakly irreducible, then $\mathscr{A}$ has a positive Perron vector. In particular, when $\mathscr{A}$ is irreducible, $\mathscr{A}$ also has a positive Perron vector; see [4-6].

In Corollary 9, a Perron vector $\mathbf{x}$ must be known in advance so that the perturbation bound can be computed. Here we derive the perturbation bound in terms of the entries of $\mathscr{A}$ only.

Lemma 12. Suppose $\mathscr{A}$ is an mth-order (m $\geq 3) n$ dimensional positive tensor. Then one has

$$
1 \leq \frac{x_{s}}{x_{t}} \leq \min _{2 \leq k, k^{\prime} \leq m} \max _{1 \leq i_{k}=i_{k}^{\prime} \leq n}\left\{\frac{\underbrace{\sum_{i_{2}, \ldots, i_{m}=1}^{n}}_{\underbrace{}_{\text {except } i_{k}}} a_{s, i_{2}, \ldots, i_{m}}}{\sum_{\underbrace{n}_{i^{\prime}}, \ldots, i_{m^{\prime}}=1}^{n} a_{t, i_{2^{\prime}}, \ldots, i_{m^{\prime}}}}\right\},
$$

where $\mathbf{x}$ is the positive Perron vector, $x_{s}=\max _{1 \leq i \leq n} x_{i}$, and $x_{t}=\min _{1 \leq i \leq n} x_{i}$.

Proof. Since $\mathscr{A}$ is a positive tensor, $\mathscr{A}$ must be irreducible and has a positive Perron vector. The left hand side of (26) is straightforward. Now we consider

$$
\begin{aligned}
\rho(\mathscr{A}) x_{t}^{m-1} & =\sum_{i_{2}, \ldots, i_{m}=1}^{n} a_{t, i_{2}, \ldots, i_{m}} x_{i_{2}} \cdots x_{i_{m}} \\
& \geq\left(\sum_{i_{2}, \ldots, i_{m}=1}^{n} a_{t, i_{2}, \ldots, i_{m}} x_{i_{k}}\right) x_{t}^{m-2},
\end{aligned}
$$

for $2 \leq k \leq n$. It implies that

$$
\rho(\mathscr{A}) x_{t} \geq \sum_{i_{2}, \ldots, i_{m}=1}^{n} a_{t, i_{2}, \ldots, i_{m}} x_{i_{k}}, \quad 2 \leq k \leq n .
$$

Similarly, we get

$$
\rho(\mathscr{A}) x_{s} \leq \sum_{i_{2}, \ldots, i_{m}=1}^{n} a_{s, i_{2}, \ldots, i_{m}} x_{i_{k}}, \quad 2 \leq k \leq n .
$$

Thus we obtain

$$
\begin{aligned}
& \frac{x_{s}}{x_{t}} \leq \frac{\sum_{i_{2}, \ldots, i_{m}=1}^{n} a_{s, i_{2}, \ldots, i_{m}} x_{i_{k}}}{\sum_{i_{2}, \ldots, i_{m}=1}^{n} a_{t, i_{2^{\prime}}, \ldots, i_{m^{\prime}}} x_{i_{k^{\prime}}}} \\
& =\frac{\sum_{i_{k}=1}^{n} \underbrace{\sum_{i_{2}, \ldots, i_{m}}^{n}=1}_{\text {except } i_{k}} a_{s, i_{2}, \ldots, i_{m}} x_{i_{k}}}{\sum_{i_{k^{\prime}}=1}^{n} \sum_{\sum_{\text {except } i_{k^{\prime}}}^{n}}^{n} a_{t, i_{2^{\prime}}, \ldots, i_{m^{\prime}}, \ldots, i_{m_{m^{\prime}}}, x_{k^{\prime}}}} .
\end{aligned}
$$

We note that, for any positive numbers $c_{i}, b_{i}$, and $y_{i}(i=$ $1,2, \ldots, n)$, we have

$$
\frac{b_{1} y_{1}+b_{2} y_{2}+\cdots+b_{n} y_{n}}{c_{1} y_{1}+c_{2} y_{2}+\cdots+c_{n} y_{n}} \leq \max _{1 \leq i \leq n} \frac{b_{i}}{c_{i}} .
$$


As $\mathscr{A}$ is a positive tensor and $\mathbf{x}$ is a positive vector, it follows from the above inequalities and (30) that

$$
\frac{x_{s}}{x_{t}} \leq \max _{1 \leq i_{k}=i_{k}^{\prime} \leq n}\left\{\frac{\underbrace{\sum_{i_{2}, \ldots, i_{m}}^{n}}_{\text {except } i_{k}}\left\{a^{a_{s, i_{2}, \ldots, i_{m}}}\right.}{\sum_{\underbrace{n}_{\text {except } i_{k^{\prime}}}}^{n} a_{t, i_{2^{\prime}}, \ldots, i_{m^{\prime}}}, \ldots, i_{m^{\prime}}=1}\right\} .
$$

Because the above upper bound is valid for $2 \leq k, k^{\prime} \leq m$, we take the minimum among them over $2 \leq k, k^{\prime} \leq m$ and we obtain the following inequality:

$$
\frac{x_{s}}{x_{t}} \leq \min _{2 \leq k, k^{\prime} \leq m} \max _{1 \leq i_{k}=i_{k}^{\prime} \leq n}\left\{\frac{\underbrace{\sum_{i_{2}, \ldots, i_{m}}^{n}=1}_{\text {except } i_{k}}{ }^{a_{s, i_{2}, \ldots, i_{m}}}}{\sum_{\underbrace{n}_{\text {except } i_{k^{\prime}}}}^{n} a^{i_{2^{\prime}}, \ldots, i_{2^{\prime}}, \ldots, i_{m^{\prime}}}}\right\} .
$$

The result follows.

Based on Lemma 12, we have the following lemma.

Lemma 13. Suppose $\mathscr{A}$ is an $m$ th-order $n$-dimensional positive tensor ( $m \geq 3$ ), and $\widetilde{\mathscr{A}}=\mathscr{A}+\Delta \mathscr{A}$ is the perturbed nonnegative tensor of $\mathscr{A}$. Then one has

$$
|\rho(\widetilde{\mathscr{A}})-\rho(\mathscr{A})| \leq \tau(\mathscr{A})\|\Delta \mathscr{A}\|_{\infty},
$$

where

$$
\begin{aligned}
& \tau(\mathscr{A}) \\
& \equiv\left(\min _{2 \leq k, k^{\prime} \leq m} \max _{1 \leq i_{k}=i_{k}^{\prime} \leq n}\left\{\frac{\underbrace{a_{s, i_{2}, \ldots, i_{m}}}_{{\text {except } i_{k}}^{i_{2}, \ldots, i_{m}}=1}}{\sum_{\underbrace{n}_{\text {except }{ }_{k^{\prime}}}}^{n} \underbrace{i_{2^{\prime}}, \ldots, i_{m^{\prime}}=1}{ }^{a_{t, i_{2^{\prime}}, \ldots, i_{m^{\prime}}}}}\right\}\right)^{m-1} .
\end{aligned}
$$

Proof. By using (25) in Corollary 9, we have

$$
\begin{aligned}
|\rho(\widetilde{\mathscr{A}})-\rho(\mathscr{A})| & \leq\left\|\Delta \mathscr{A} \times{ }_{1} D_{\mathbf{x}}^{-(m-1)} \times{ }_{2} D_{\mathbf{x}} \times{ }_{3} \cdots \times{ }_{m} D_{\mathbf{x}}\right\|_{\infty} \\
& \leq\left\|D_{\mathbf{x}}^{-(m-1)}\right\|_{\infty}\left\|D_{\mathbf{x}}^{m-1}\right\|_{\infty}\|\Delta \mathscr{A}\|_{\infty} .
\end{aligned}
$$

The result follows.

Remark 14. The perturbation bound in Lemma 13 can be achieved by considering $\mathscr{A}=\mathscr{E}$ and $\widetilde{\mathscr{A}}=\mathscr{E}+\mathcal{E} \mathscr{I}$, where $\varepsilon$ is a positive real number and $\mathscr{E}$ is a tensor with all the entries being equal to one. It is clear that $\mathscr{E}$ is irreducible; that is, $\mathscr{E}$ has the unique positive Perron vector. And we just note that the largest eigenvalues of $\mathscr{E}$ and $\mathscr{E}+\mathcal{E} \mathscr{I}$ are equal to $n^{m-1}$ and $n^{m-1}+\varepsilon$, respectively, $\|\Delta \mathscr{A}\|_{\infty}=\varepsilon$, and $\tau(\mathscr{E})=1$.

Corollary 15. Suppose $\mathscr{A}$ is an mth-order $n$-dimensional nonnegative tensor, and $\widetilde{\mathscr{A}}=\mathscr{A}+\Delta \mathscr{A}$ is the perturbed positive tensor of $\mathscr{A}$. Then one has

$$
|\rho(\widetilde{\mathscr{A}})-\rho(\mathscr{A})| \leq \tau(\widetilde{\mathscr{A}})\|\Delta \mathscr{A}\|_{\infty},
$$

where $\tau(\cdot)$ is defined in (35).
Proof. We just switch the roles of $\mathscr{A}$ and $\widetilde{\mathscr{A}}$ in Lemma 13.

Let us state the following lemma to handle the case when $\mathscr{A}$ is a nonnegative tensor.

Lemma 16. Suppose $\mathscr{A}$ is an mth-order $n$-dimensional nonnegative tensor, and $\mathscr{A}_{k}=\mathscr{A}+(1 / k) \mathscr{E}$. Then $\rho(\mathscr{A})=$ $\lim _{k \rightarrow \infty} \rho\left(\mathscr{A}_{k}\right)$.

The proof of this lemma is similar to Theorem 2.3 given in [7].

Suppose that $\mathscr{A}$ is a nonnegative tensor. Let $\mathscr{A}_{k}=\mathscr{A}+$ $(1 / k) \mathscr{J}$. It is clear that $\mathscr{A}_{k}$ is a positive tensor. Let $\widetilde{\mathscr{A}}_{k}=\mathscr{A}_{k}+$ $\Delta \mathscr{A}$ be a perturbed nonnegative tensor of $\mathscr{A}_{k}$. By applying Corollary 15 to $\mathscr{A}_{k}$ and $\widetilde{\mathscr{A}}_{k}$, we have

$$
\left|\rho\left(\widetilde{\mathscr{A}}_{k}\right)-\rho\left(\mathscr{A}_{k}\right)\right| \leq \tau\left(\mathscr{A}_{k}\right)\|\Delta \mathscr{A}\|_{\infty} .
$$

Therefore, when $k \rightarrow \infty$, by Lemma 16, we have the following theorem.

Theorem 17. Suppose $\mathscr{A}$ is an mth-order n-dimensional nonnegative tensor, and $\widetilde{\mathscr{A}}=\mathscr{A}+\Delta \mathscr{A}$ is the perturbed nonnegative tensor of $\mathscr{A}$. Then one has

$$
|\rho(\widetilde{\mathscr{A}})-\rho(\mathscr{A})| \leq \tau(\mathscr{A})\|\Delta \mathscr{A}\|_{\infty}
$$

provided that $\tau(\mathscr{A})>0$.

Example 18. We conduct an experiment to verify the perturbation bound. We randomly construct a positive tensor $\mathscr{A}$, where each entry is generated by uniform distribution $[0,1]$. We further check the value of each entry must be greater than zero, and therefore the constructed tensor is positive. In the experiment, $\mathscr{A}$ is perturbed to a positive tensor $\widetilde{\mathscr{A}}$ by adding $\varepsilon \Delta \mathscr{A}$, where $\varepsilon$ is a positive number and $\Delta \mathscr{A}$ is a positive tensor randomly generated by the above-mentioned method. We study the absolute difference $|\rho-\tilde{\rho}|$ between the spectral radii of $\mathscr{A}$ and $\widetilde{\mathscr{A}}$ and the perturbation bound in Corollary 9. In Figure 1, we show the results for $n=$ 5,10 and $m=4$. For each point in the figure, we give the average value of $|\rho-\tilde{\rho}|,\left\|\Delta \mathscr{A} \times{ }_{1} D_{\mathbf{x}}^{-(m-1)} \times{ }_{2} D_{\mathbf{x}} \times{ }_{3} \cdots \times{ }_{m} D_{\mathbf{x}}\right\|_{\infty}$, or $\tau(\mathscr{A})\|\Delta \mathscr{A}\|_{\infty}$ based on the computed results for 100 randomly constructed positive tensors. The $x$-axis refers to values of $\varepsilon$ : $0.01,0.005,0.001,0.0005,0.0001$, and 0.00005 . We see from the figures that the average values (in logarithm scale) depend linearly on $\varepsilon$ (in logarithm scale). The perturbation bounds $\left\|\Delta \mathscr{A} \times_{1} D_{\mathbf{x}}^{-(m-1)} \times_{2} D_{\mathbf{x}} \times_{3} \cdots \times_{m} D_{\mathbf{x}}\right\|_{\infty}$ and $\tau(\mathscr{A})\|\Delta \mathscr{A}\|_{\infty}$ provide the upper bound of $|\rho-\tilde{\rho}|$. This result is consistent with our prediction in the theory. It is interesting to note that the bound $\left\|\Delta \mathscr{A} D_{\mathbf{x}}^{-(m-1)} \times_{2} D_{\mathbf{x}} \times_{3} \cdots \times_{m} D_{\mathbf{x}}\right\|_{\infty}$ is very close to the actual difference.

2.1. Application to the NQZ Algorithm. In this subsection, we apply our perturbation results to the NQZ algorithm [10] which is an iterative method for finding the spectral radius of a nonnegative tensor. The NQZ algorithm presented in [10] is given as follows. 


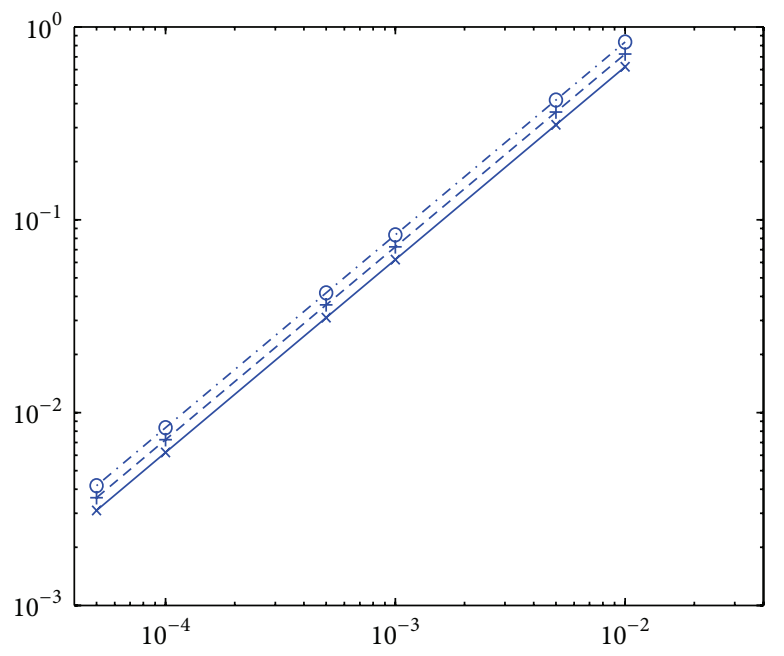

(a)

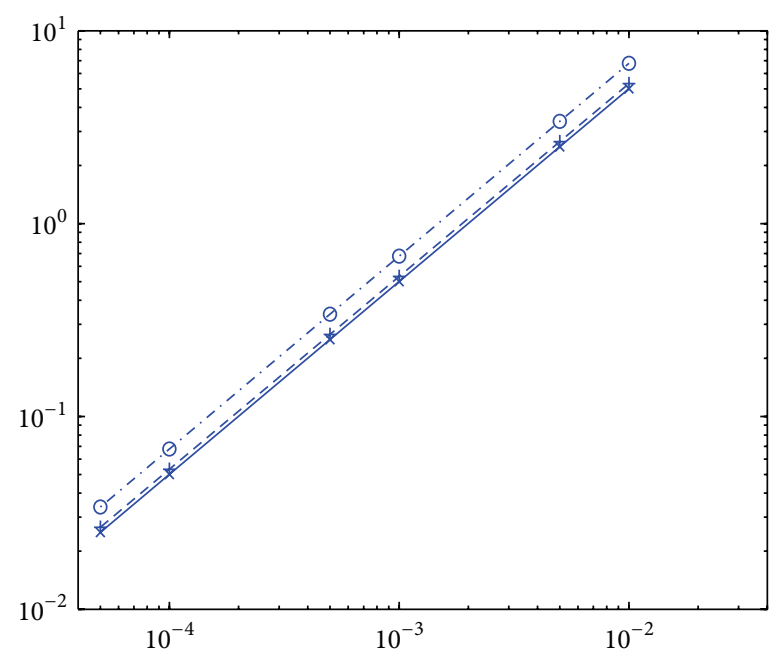

(b)

FIGURE 1: The results of the perturbation bounds (a) $n=5$ and $m=4$ and (b) $n=10$ and $m=4:-|\lambda-\tilde{\lambda}|$ (the actual difference); - - $\left\|\Delta \mathscr{A} D_{\mathbf{x}}^{-(m-1)} \times_{2} D_{\mathbf{x}} \times_{3} \cdots \times{ }_{m} D_{\mathbf{x}}\right\|_{\infty}$ and $-\cdot-\cdot \cdot-\cdot \tau(\mathscr{A})\|\Delta \mathscr{A}\|_{\infty}$.

Choose $\mathbf{u}^{(0)} \in \operatorname{int}(\mathbb{P})$ and let $\mathbf{v}=\mathscr{A}\left(\mathbf{u}^{(0)}\right)^{m-1}$. For $k=$ $0,1,2, \ldots$, compute

$$
\begin{array}{cc}
\mathbf{u}^{(k)}=\frac{\left(\mathbf{v}^{(k)}\right)^{[1 /(m-1)]}}{\|\left(\mathbf{v}^{(k)}\right)^{[1 /(m-1)]}} ; & \mathbf{v}^{(k+1)}=\mathscr{A}\left(\mathbf{u}^{(k+1)}\right)^{m-1} ; \\
\underline{\lambda}_{k+1}=\min _{u_{i}^{(k+1)}>0} \frac{v_{i}^{(k+1)}}{\left(u_{i}^{(k+1)}\right)^{m-1}} ; & \bar{\lambda}_{k+1}=\max _{u_{i}^{(k+1)}>0} \frac{v_{i}^{(k+1)}}{\left(u_{i}^{(k+1)}\right)^{m-1}} .
\end{array}
$$

It is shown in $[10]$ that the sequences $\{\underline{\lambda}\}$ and $\{\bar{\lambda}\}$ converge to some numbers $\underline{\lambda}$ and $\bar{\lambda}$, respectively, and we have $\underline{\lambda} \leq$ $\rho(\mathscr{A}) \leq \bar{\lambda}$. If $\underline{\lambda}=\bar{\lambda}$, the gap is zero and therefore both the sequences $\{\underline{\lambda}\}$ and $\{\bar{\lambda}\}$ converge to $\rho(\mathscr{A})$. However, a positive gap may happen, which can be seen in an example given in [10]. Pearson [13] introduced the notion of essentially positive tensors and showed the convergence of the NQZ algorithm for essentially positive tensors. Chang et al. [8] further established the convergence of the NQZ algorithm for primitive tensors.

Definition 19. An $m$ th-order $n$-dimensional tensor $\mathscr{A}$ is essentially positive if $\mathscr{A} \mathbf{y}^{m-1} \in \operatorname{int}(\mathbb{P})$ for any nonzero $\mathbf{y} \in \mathbb{P}$.

Definition 20. An $m$ th-order $n$-dimensional tensor $\mathscr{A}$ is called primitive if there exists a positive integer $k$ such that $\mathscr{A}^{k} \mathbf{y} \in \operatorname{int}(\mathbb{P})$ for any nonzero $\mathbf{y} \in \mathbb{P}$.

An essentially positive tensor is a primitive tensor, and a primitive tensor is an irreducible nonnegative tensor but not vice versa.

Now we demonstrate how to use the results in Theorem 7 to estimate the spectral radius of a nonnegative tensor via the NQZ algorithm. For example, when $\mathscr{A}$ is a reducible nonnegative tensor, then the NQZ algorithm may not be convergent. Our idea is to take a small perturbation $\Delta \mathscr{A}=$ $\mathcal{E} \mathcal{F}$, where $\mathcal{\varepsilon}$ is a very small positive number and $\mathscr{J}$ is a tensor with all the entries being equal to one. It is clear that $\widetilde{\mathscr{A}}=$ $\mathscr{A}+\varepsilon \mathscr{J}$ is essentially positive. Therefore, when we apply the NQZ algorithm to compute the largest eigenvalue of $\widetilde{\mathscr{A}}$, it is convergent. Without loss of generality, we normalize the output vector $\mathbf{u}$ in the NQZ algorithm. In particular, the 1norm of $\mathbf{u}$ is employed. The output of the algorithm contains a positive number $\tilde{\lambda}$ and $\mathbf{u}>0$ with $\|\mathbf{u}\|_{1}=1$ (i.e., $\sum_{i=1}^{n} u_{i}=1$ ). We note that

$$
\begin{aligned}
& \left(\varepsilon \mathscr{J} D_{\mathbf{u}}^{-(m-1)} \times{ }_{2} D_{\mathbf{u}} \times{ }_{3} \cdots \times \times_{m} D_{\mathbf{u}} \mathbf{e}^{m-1}\right)_{i_{1}} \\
& =\sum_{i_{2}, \ldots, i_{m}=1}^{n} \frac{(\varepsilon \mathcal{J})_{i_{1}, i_{2}, \ldots, i_{m}} u_{i_{2}} \cdots u_{i_{m}}}{u_{i_{1}}^{m-1}} \\
& =\frac{\varepsilon}{u_{i_{1}}^{m-1}} \sum_{i_{2}, \ldots, i_{m}=1}^{n} u_{i_{2}} \cdots u_{i_{m}} \\
& =\frac{\varepsilon}{u_{i_{1}}^{m-1}} .
\end{aligned}
$$

Then, by Theorem 7, we have

$$
\frac{\varepsilon}{\max _{1 \leq i \leq n} u_{i}^{m-1}} \leq \rho(\widetilde{\mathscr{A}})-\rho(\mathscr{A}) \leq \frac{\varepsilon}{\min _{1 \leq i \leq n} u_{i}^{m-1}}
$$

That is,

$$
\rho(\widetilde{\mathscr{A}})-\frac{\varepsilon}{\min _{1 \leq i \leq n} u_{i}^{m-1}} \leq \rho(\mathscr{A}) \leq \rho(\widetilde{\mathscr{A}})-\frac{\varepsilon}{\max _{1 \leq i \leq n} u_{i}^{m-1}} .
$$


Let $u_{s}=\max _{1 \leq i \leq n} u_{i}$ and $u_{t}=\min _{1 \leq i \leq n} u_{i}$. By considering $m \geq 3$, we know that

$$
\begin{aligned}
& \rho(\widetilde{\mathscr{A}}) u_{t}^{m-1} \\
& =\sum_{i_{2}, \ldots, i_{m}=1}^{n}(\mathscr{A}+\mathcal{E} \mathscr{J})_{t, i_{2}, \ldots, i_{m}} u_{i_{2}} \cdots u_{i_{m}} \\
& \geq u_{t}^{m-2} \max _{2 \leq k \leq m}\left\{\begin{array}{l}
\sum_{\text {except } i_{k}}^{n}(\mathscr{A}+\varepsilon \mathcal{E})_{t, i_{2}, \ldots, i_{m}} u_{i_{k}} \\
\sum_{2}^{i_{2}, \ldots, i_{m}=1}
\end{array}\right\} \\
& \geq u_{t}^{m-2} \max _{2 \leq k \leq m}\{\min _{1 \leq i_{k} \leq n} \underbrace{\sum_{i_{2}, \ldots, i_{m}}^{n}=1}_{\underbrace{}_{\text {except } i_{k}}}(\mathscr{A}+\mathcal{E} \mathscr{J})_{t, i_{2}, \ldots, i_{m}} \sum_{i_{k}=1}^{n} u_{i_{k}}\} \\
& =u_{t}^{m-2} \max _{2 \leq k \leq m}\{\min _{1 \leq i_{k} \leq n} \underbrace{\sum_{i_{2}, \ldots, i_{m}=1}^{n}(\mathscr{A}+\mathcal{E} \mathcal{J})_{t, i_{2}, \ldots, i_{m}}}_{\text {except } i_{k}}\} \\
& =u_{t}^{m-2}(\varepsilon n^{m-2}+\max _{2 \leq k \leq m} \min _{1 \leq i_{k} \leq n} \underbrace{n}_{\underbrace{i_{2}, \ldots, i_{m}=1}_{\text {except } i_{k}}} a_{t, i_{2}, \ldots, i_{m}}) ;
\end{aligned}
$$

that is, we have

$$
\rho(\widetilde{\mathscr{A}}) u_{t} \geq \varepsilon n^{m-2}+\max _{2 \leq k \leq m} \min _{1 \leq i_{1}, i_{k} \leq n} \underbrace{\sum_{i_{2}, \ldots, i_{m}}^{n} a_{i_{1}, i_{2}, \ldots, i_{m}}}_{\text {except } i_{k}}
$$

or

$$
\frac{1}{u_{t}} \leq \frac{\rho(\widetilde{\mathscr{A}})}{\varepsilon n^{m-2}+\max _{2 \leq k \leq m} \min _{1 \leq i_{1}, i_{k} \leq n} \underbrace{\sum_{i_{2}, \ldots, i_{m}=1}^{n}}_{\text {except } i_{k}} a_{i_{1}, i_{2}, \ldots, i_{m}}} .
$$

On the other hand,

$$
\begin{aligned}
\rho(\widetilde{\mathscr{A}}) u_{s}^{m-1} & =\sum_{i_{2}, \ldots, i_{m}=1}^{n}(\mathscr{A}+\mathcal{E} \mathscr{J})_{s, i_{2}, \ldots, i_{m}} u_{i_{2}} \cdots u_{i_{m}} \\
& \leq \sum_{i_{2}, \ldots, i_{m}=1}^{n}(\mathscr{A}+\mathcal{E} \mathscr{J})_{s, i_{2}, \ldots, i_{m}} \leq \varepsilon n^{m-1}+\|\mathscr{A}\|_{\infty} .
\end{aligned}
$$

By putting (46) and (47) into (43), we have

$$
\begin{aligned}
& \rho(\widetilde{\mathscr{A}})\left(1-\frac{\varepsilon \rho(\widetilde{\mathscr{A}})^{m-2}}{(\varepsilon n^{m-2}+\max _{2 \leq k \leq m} \min _{1 \leq i_{1}, i_{k} \leq n} \underbrace{i_{i_{1}, i_{2}, \ldots, i_{m}}}_{i_{i_{2}, \ldots, i_{m}}^{n} i_{i_{k}}})^{m-1}}\right) \\
& \leq \rho(\mathscr{A}) \leq \rho(\widetilde{\mathscr{A}})\left(1-\frac{\varepsilon}{\varepsilon n^{m-1}+\|\mathscr{A}\|_{\infty}}\right) .
\end{aligned}
$$

One may know $\rho(\mathscr{A})=\widetilde{\lambda}+O(\varepsilon)$ if

$$
\max _{2 \leq k \leq m} \min _{1 \leq i_{1}, i_{k} \leq n} \underbrace{n}_{\text {except } i_{k}} \sum_{i_{1}, i_{2}, \ldots, i_{m}}^{n}>0
$$

This shows that we can estimate $\rho(\mathscr{A})$ of a nonnegative tensor $\mathscr{A}$ with a specified precision via the NQZ algorithm for the computation of $\rho(\widetilde{\mathscr{A}})$.
Remark 21. Liu et al. [9] modified the NQZ algorithm for computing the spectral radius of $\mathscr{A}+\mathcal{E} \mathscr{I}$, where $\mathscr{A}$ is an irreducible nonnegative tensor and $\varepsilon$ is a very small number, and showed that the algorithm converges to $\rho(\mathscr{A})+\varepsilon$. This fact can be explained by our theoretical analysis in Theorem 7 by setting $\Delta A=\varepsilon \mathscr{I}$. We just note that the left and right sides of the inequality in (15) are equal to $\varepsilon$; that is, $\varepsilon \leq$ $\rho(\widetilde{\mathscr{A}})-\rho(\mathscr{A}) \leq \varepsilon$. It implies that $\rho(\widetilde{\mathscr{A}})=\rho(\mathscr{A})+\varepsilon$. However, when the given nonnegative tensor is not irreducible, then their results cannot be valid. However, our approach can still be used to estimate the spectral radius of a nonnegative tensor in general. 
TABLE 1: Numerical results for different $\varepsilon$ in Example 22.

\begin{tabular}{lccr}
\hline$\varepsilon$ & $\rho(\widetilde{\mathscr{A}})-\frac{\varepsilon}{\min _{1 \leq i \leq n} u_{i}^{m-1}}$ & $\rho(\widetilde{\mathscr{A}})-\frac{\varepsilon}{\max _{1 \leq i \leq n} u_{i}^{m-1}}$ & Difference \\
\hline $1 e-2$ & 1.399817488643705 & 1.428757688931172 & 0.028940200287467 \\
$1 e-3$ & 1.412729187546902 & 1.415699496853463 & 0.002970309306561 \\
$1 e-4$ & 1.414064662464100 & 1.414362477963432 & 0.000297815499332 \\
$1 e-5$ & 1.414198667753479 & 1.414228457171375 & 0.000029789417896 \\
$1 e-6$ & 1.414212073004730 & 1.414215052025221 & 0.000002979020491 \\
\hline
\end{tabular}

Example 22. In [8], the following 3rd order 3-dimensional nonnegative tensor is considered: $a_{1,2,2}=a_{1,3,3}=a_{2,1,1}=$ $a_{3,1,1}=1$ and the other entries are equal to zero. It can be shown that this tensor $\mathscr{A}$ is irreducible, but not primitive. There is no convergence result of the NQZ algorithm for such $\mathscr{A}$, and the spectral radius of $\mathscr{A}$ is equal to $\sqrt{2} \approx$ 1.414213562373095; see [8]. In this example, we take the perturbation $\Delta \mathscr{A}=\varepsilon \mathscr{J}$. We apply the NQZ algorithm to compute the spectral radius of $\widetilde{\mathscr{A}}$ to approximate the actual one. According to Table 1 , the results show that $\rho(\mathscr{A})$ is about $\rho(\widetilde{\mathscr{A}})+O(\varepsilon)$.

Example 23. We consider the following 3rd order 3dimensional nonnegative tensor: $a_{1,1,1}=a_{1,2,2}=a_{1,3,3}=$ $a_{2,1,1}=a_{2,2,2}=a_{2,3,3}=a_{3,1,1}=a_{3,2,2}=a_{3,3,3}=1$ and the other entries are equal to zero. It can be shown that this tensor $\mathscr{A}$ is reducible. The spectral radius of $\mathscr{A}$ is equal to 3 . There is no convergence result of the NQZ algorithm for such reducible nonnegative tensor. It is interesting to note that this tensor satisfies (49). In this example, we take the perturbation $\Delta \mathscr{A}=$ $\varepsilon \mathscr{F}$. The spectral radii of $\widetilde{\mathscr{A}}$ by the NQZ algorithm are still accurate approximations of $\rho(\mathscr{A}): 3.090000000000000(\varepsilon=$ 0.01), $3.009000000000000(\varepsilon=0.001), 3.000900000000000$ $(\varepsilon=0.0001), 3.000090000000001(\varepsilon=0.00001)$, and $3.000009000000000(\varepsilon=0.000001)$. Indeed, both the values in the left and right sides of (43) are equal to 3.000000000000000 (up to the number of decimals shown in MATLAB). These results show the bounds are very tight and $\rho(\mathscr{A})$ is about $\rho(\widetilde{\mathscr{A}})+O(\varepsilon)$.

Example 24. Now we consider the following 3rd order 3dimensional nonnegative tensor: $a_{1,1,1}=1$ and the other entries are equal to zero. $\mathscr{A}$ is a reducible nonnegative tensor. For such $\mathscr{A}$, we know the $a$ is the spectral radius of $\mathscr{A}$ and $[1,0, \ldots, 0]^{T}$ is the corresponding eigenvector (the normalized in 2-norm). In this example, we take the perturbation $\Delta \mathscr{A}=\varepsilon \mathscr{J}$. Although this tensor does not satisfy (49), the spectral radii of $\widetilde{\mathscr{A}}$ by the NQZ algorithm are still accurate approximate of $\rho(\mathscr{A}): 1.015565072567277(\varepsilon=$ $0.01), 1.001139501996208(\varepsilon=0.001), 1.000104123060726$ $(\varepsilon=0.0001), 1.000010127700654(\varepsilon=0.00001)$, and $1.000001004012030(\varepsilon=0.000001)$. We find that the values in the left and right sides of (43) are close to 1 and 0 , respectively, for different values of $\varepsilon$. It is clear that the bounds are not tight. It is interesting to note that the maximum and minimum values of the entries of the associated eigenvector are 1 and 0 ; we expect that the error bound in (43) can be very poor. Indeed, the errors between the actual eigenvector and the approximate eigenvector are $0.243064619277186(\varepsilon=$ $0.01), 0.077415571882820(\varepsilon=0.001), 0.024493622285564$ $(\varepsilon=0.0001), 0.007745927468297(\varepsilon=0.00001)$, and $0.002449488513126(\varepsilon=0.000001)$. Thus the approximation is more accurate for the largest eigenvalue than for the associated eigenvector.

\section{Backward Error Bound}

In this section, we study the backward error tensor $\Delta \mathscr{A}$ and obtain its smallest error bound of $\Delta \mathscr{A}$ such that

$$
\widetilde{\mathscr{A}} \widetilde{\mathbf{x}}^{m-1}=(\mathscr{A}+\Delta \mathscr{A}) \widetilde{\mathbf{x}}^{m-1}=\widetilde{\lambda} \widetilde{\mathbf{x}}^{[m-1]},
$$

where $\mathscr{A}$ and $\widetilde{\mathscr{A}}=\mathscr{A}+\Delta \mathscr{A}$ are irreducible nonnegative tensors with $\Delta \mathscr{A}=\left(\delta a_{i_{1}, i_{2}, \ldots, i_{m}}\right)$ for $1 \leq i_{1}, i_{2}, \ldots, i_{m} \leq n, \tilde{\lambda}$ is the largest eigenvalue of $\widetilde{\mathscr{A}}$, and $\widetilde{\mathbf{x}}$ is a Perron vector of $\widetilde{\mathscr{A}}$. The backward error for the eigenpair is defined as follows:

$$
\eta(\widetilde{\mathbf{x}})=\min _{\Delta \mathscr{A} \in \Omega}\|\Delta \mathscr{A}\|_{F}
$$

where

$$
\begin{gathered}
\Omega=\{\Delta \mathscr{A} \text { is a nonnegative tensor such that } \\
\mathscr{A}+\Delta \mathscr{A} \text { is irreducible }\}
\end{gathered}
$$

and $\|\Delta \mathscr{A}\|_{F}:=\sqrt{\sum_{i_{1}, \ldots, i_{m}=1}^{n} \delta a_{i_{1}, \ldots, i_{m}}^{2}}$.

Before we show the results of the backward error in the computation of the largest eigenvalue of an irreducible nonnegative tensor, we need the following lemma given in [14, Theorem 9.1].

Lemma 25. Let $U \in \mathbb{C}^{p \times q}, V \in \mathbb{C}^{r \times s}$, and $W \in \mathbb{C}^{p \times s}$. Let

$$
\begin{aligned}
& \mathbb{X}=\left\{X \in \mathbb{C}^{p \times s}: U X V=W\right\}, \\
& \mathbb{Y}=\left\{U^{\dagger} W V^{\dagger}+Y-P_{U^{*}} Y P_{V}\right\} .
\end{aligned}
$$

Then

$$
P_{U} W P_{V^{*}}=W
$$

if and only if $\mathbb{X} \neq \emptyset$, and when $\mathbb{\emptyset} \neq \emptyset, \mathbb{X}=\mathbb{Y}$, where $\cdot{ }^{*}$ denotes the conjugate transpose of a matrix, $U^{\dagger}$ is the Moore-Penrose generalized inverse of $U, P_{U}=U U^{\dagger}$, and $P_{V^{*}}=V^{\dagger} V$. 
Now we show the main result of this section.

Theorem 26. Suppose $\mathscr{A}$ is an mth-order $n$-dimensional irreducible nonnegative tensor. Let $\bar{\lambda}$ and $\mathbf{u}$ be the output of the NQZ algorithm (stated in Section 2.1) applied to $\mathscr{A}$, and $\mathbf{r}=\bar{\lambda} \mathbf{u}^{[m-1]}-\mathscr{A} \mathbf{u}^{m-1}$. Then the backward error for $\bar{\lambda}$ and $\mathbf{u}$ of the perturbed irreducible nonnegative tensor $\mathscr{A}+\Delta \mathscr{A}$ is given by

$$
\eta(\mathbf{u})=\frac{\|\mathbf{r}\|_{2}}{\|\mathbf{u}\|_{2}^{m-1}}
$$

and $\Delta \mathscr{A}$ can be chosen as follows:

$$
\delta a_{i_{1}, i_{2}, \ldots, i_{m}}=\frac{(\mathbf{r})_{i_{1}} \cdot(\mathbf{u})_{i_{2}} \cdots(\mathbf{u})_{i_{m}}}{\|\mathbf{u}\|_{2}^{2(m-1)}} .
$$

Proof. We note that

$$
\Delta \mathscr{A} \mathbf{u}^{m-1}=\bar{\lambda} \mathbf{u}^{[m-1]}-\mathscr{A} \mathbf{u}^{m-1}=\mathbf{r} .
$$

In order to analyze $\mathbf{u}$, we rewrite the above equation into a linear system as follows:

$$
\Delta A \mathbf{b}=\Delta A(\overbrace{\mathbf{u} \otimes \cdots \otimes \mathbf{u}}^{m-1})=\mathbf{r},
$$

where $\Delta A$ is an $n$-by- $n^{m-1}$ matrix with its entries given by

$$
\begin{array}{r}
(\Delta A)_{i_{1}, j}=\delta a_{i_{1}, i_{2}, \ldots, i_{m}} \text { with } j=i_{2} \times \cdots \times i_{m}, \\
1 \leq i_{1}, i_{2}, \ldots, i_{m} \leq n .
\end{array}
$$

It follows from Lemma 25 that

$$
\Delta A=\mathbf{r b}^{\dagger}+Z\left(I-\mathbf{b}^{\dagger}\right)
$$

where $\mathbf{b}^{\dagger}$ is an 1 -by- $n^{m-1}$ vector given by $\mathbf{b} /\|\mathbf{b}\|_{2}$ and $Z$ is an $n$-by- $n^{m-1}$ matrix. Therefore, we obtain

$$
\|\Delta A\|_{F}^{2}=\left\|\mathbf{r b}^{\dagger}\right\|_{F}^{2}+\left\|Z\left(I-\mathbf{b}^{\dagger}\right)\right\|_{F}^{2}
$$

The minimization of $\|\Delta A\|_{F}^{2}$ is equivalent to finding a matrix $Z$ such that $\left\|\mathbf{r b}^{\dagger}\right\|_{F}^{2}+\left\|Z\left(I-\mathbf{b b}^{\dagger}\right)\right\|_{F}^{2}$ can be minimized. It is clear that we choose $Z=0$; that is, $\min \|\Delta A\|_{F}^{2}=\left\|\mathbf{r} \mathbf{b}^{\dagger}\right\|_{F}^{2}$.

According to the NQZ algorithm stated in Section 2.1, we know that $\mathbf{r}$ is nonnegative. As $\mathbf{u}$ is positive, both $\mathbf{b}$ and $\mathbf{b}^{\dagger}$ are also positive. Let us consider two cases. We note that if $\mathbf{r}$ is a zero vector, then $\bar{\lambda}$ is the largest eigenvalue of $\mathscr{A}$, and the problem is solved. Now we consider $\mathbf{r}$ is not a zero vector. It follows that at least one row of $\Delta A=\mathbf{r b}^{\dagger}$ must be positive. It can be shown that $\widetilde{\mathscr{A}}$ is irreducible. The argument is that for any nonempty proper index subset $\mathbb{\complement} \subset\{1,2, \ldots, n\}$ such that

$$
a_{i_{1}, i_{2}, \ldots, i_{m}}+\delta_{i_{1}, i_{2}, \ldots, i_{m}}>0, \quad \forall i_{1} \in \mathbb{J}, \forall i_{2}, \ldots, i_{m} \notin \mathbb{J} .
$$

Hence the result follows.
TABLE 2: The backward errors for different cases.

\begin{tabular}{lcc}
\hline$m$ & $n$ & Backward error $\eta(\widetilde{\mathbf{x}})$ \\
\hline 3 & 5 & $1.2297 e-15$ \\
3 & 10 & $5.5960 e-15$ \\
3 & 20 & $1.5579 e-14$ \\
3 & 40 & $5.9253 e-14$ \\
\hline 4 & 5 & $9.9174 e-15$ \\
4 & 10 & $6.4772 e-14$ \\
4 & 20 & $4.3210 e-13$ \\
4 & 40 & $2.9931 e-12$ \\
\hline
\end{tabular}

Remark 27. When $m=2$, the results of the backward error are reduced to the results in Theorem 6.3.2 in [14]; that is,

$$
\eta(\widetilde{\mathbf{x}})=\left\|\mathbf{r} \widetilde{\mathbf{x}}^{\dagger}\right\|_{F} \cdot
$$

Example 28. Let us conduct an experiment to check the backward error when we use the NQZ algorithm for computing the largest eigenvalue of an irreducible nonnegative tensor. We randomly construct a positive tensor $\mathscr{A}$, where each entry is generated by uniform distribution $[0,1]$. We further check the value of each entry must be greater than zero, and therefore the constructed tensor is positive. In Table 2, we show the average backward error based on the computed results for 100 randomly constructed positive tensors. We see from the table that the backward error can be very small, but it still depends on the order $m$ of and the dimension $n$ of the tensor. The backward error is large when $m$ or $n$ is large.

\section{Concluding Remarks}

In summary, we have studied and derived the perturbation bounds for the spectral radius of a nonnegative tensor. Numerical examples have been given to demonstrate our theoretical results. Also we have investigated the backward error matrix $\Delta \mathscr{A}$ and obtain its smallest error bound for its perturbed largest eigenvalue and associated eigenvector of an irreducible nonnegative tensor. Numerical examples have shown that the backward error can be very small. These results may show that the NQZ algorithm may be backward stable for the computation of the largest eigenvalue of an irreducible nonnegative tensor.

In this paper, we do not study the perturbation bound of the eigenvector corresponding to the largest eigenvalue of a nonnegative tensor. This would be an interesting research topic for future consideration.

\section{Conflict of Interests}

The authors declare that there is no conflict of interests regarding the publication of this paper.

\section{Acknowledgments}

The authors thank the referee for his/her valuable suggestions. Wen Li was supported in part by National Natural Science Foundations of China (nos. 10971075, 11271144), 
Guangdong Provincial Natural Science Foundations (no. s2012010009985), and Project of Department of Education of Guangdong Province (no. 2013KJCX0053). Research was supported in part by RGC GRF Grant no. 201812.

\section{References}

[1] X. Li, M. K. Ng, and Y. Ye, "HAR: hub, authority and relevance scores in multi-relational data for query search," in Proceedings of the 12th SIAM International Conference on Data Mining (SDM '12), pp. 141-152, Anaheim, Calif, USA, April 2012.

[2] M. K. Ng, X. Li, and Y. Ye, "MultiRank: co-ranking for objects and relations in multi-relational data," in Proceedings of the 17th ACM SIGKDD International Conference on Knowledge Discovery and Data Mining (KDD '11), pp. 1217-1225, August 2011.

[3] L. Qi, "Eigenvalues of a real supersymmetric tensor," Journal of Symbolic Computation, vol. 40, no. 6, pp. 1302-1324, 2005.

[4] L.-H. Lim, "Singular values and eigenvalues of tensors: a variational approach," in Proceedings of the 1st IEEE International Workshop on Computational Advances in Multi-Sensor Adaptive Processing (CAMSAP '05), pp. 129-132, Puerto Vallarta, Mexico, December 2005.

[5] K. C. Chang, K. Pearson, and T. Zhang, "Perron-Frobenius theorem for nonnegative tensors," Communications in Mathematical Sciences, vol. 6, no. 2, pp. 507-520, 2008.

[6] S. Friedland, S. Gaubert, and L. Han, "Perron-Frobenius theorem for nonnegative multilinear forms and extensions," Linear Algebra and its Applications, vol. 438, no. 2, pp. 738-749, 2013.

[7] Y. Yang and Q. Yang, "Further results for Perron-Frobenius theorem for nonnegative tensors," SIAM Journal on Matrix Analysis and Applications, vol. 31, no. 5, pp. 2517-2530, 2010.

[8] K.-C. Chang, K. J. Pearson, and T. Zhang, "Primitivity, the convergence of the NQZ method, and the largest eigenvalue for nonnegative tensors," SIAM Journal on Matrix Analysis and Applications, vol. 32, no. 3, pp. 806-819, 2011.

[9] Y. Liu, G. Zhou, and N. F. Ibrahim, "An always convergent algorithm for the largest eigenvalue of an irreducible nonnegative tensor," Journal of Computational and Applied Mathematics, vol. 235, no. 1, pp. 286-292, 2010.

[10] M. Ng, L. Qi, and G. Zhou, "Finding the largest eigenvalue of a nonnegative tensor," SIAM Journal on Matrix Analysis and Applications, vol. 31, no. 3, pp. 1090-1099, 2009.

[11] L. de Lathauwer, B. de Moor, and J. Vandewalle, "A multilinear singular value decomposition," SIAM Journal on Matrix Analysis and Applications, vol. 21, no. 4, pp. 1253-1278, 2000.

[12] S. L. Liu and S. Y. Wang, "Sensitivity analysis of nonnegative irreducible matrices," Applied Mathematics Letters, vol. 12, no. 2, pp. 121-124, 1999.

[13] K. Pearson, "Essentially positive tensors," International Journal of Algebra, vol. 4, pp. 421-427, 2010.

[14] J. Sun, Matrix Perturbation Analysis, Science Press, Beijing, China, 2001. 


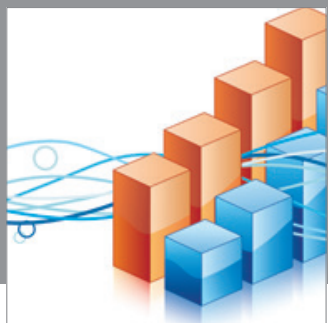

Advances in

Operations Research

mansans

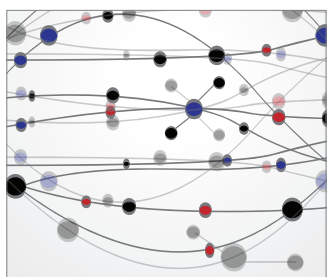

The Scientific World Journal
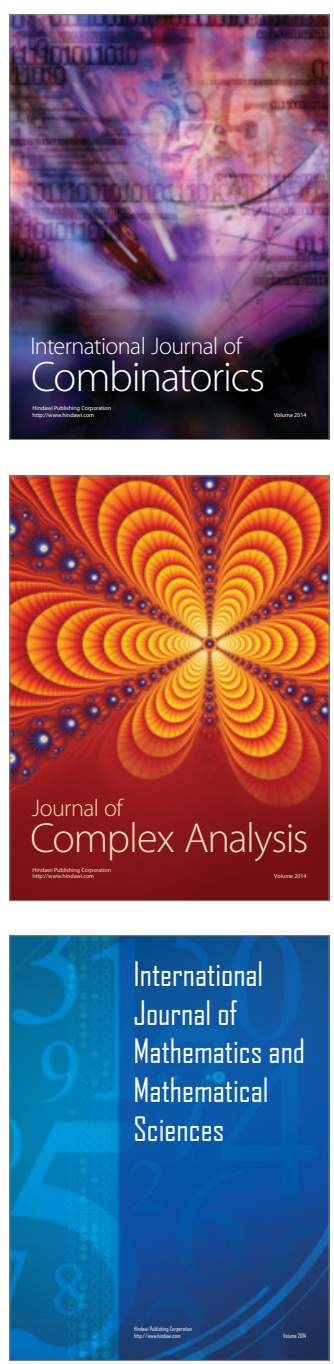
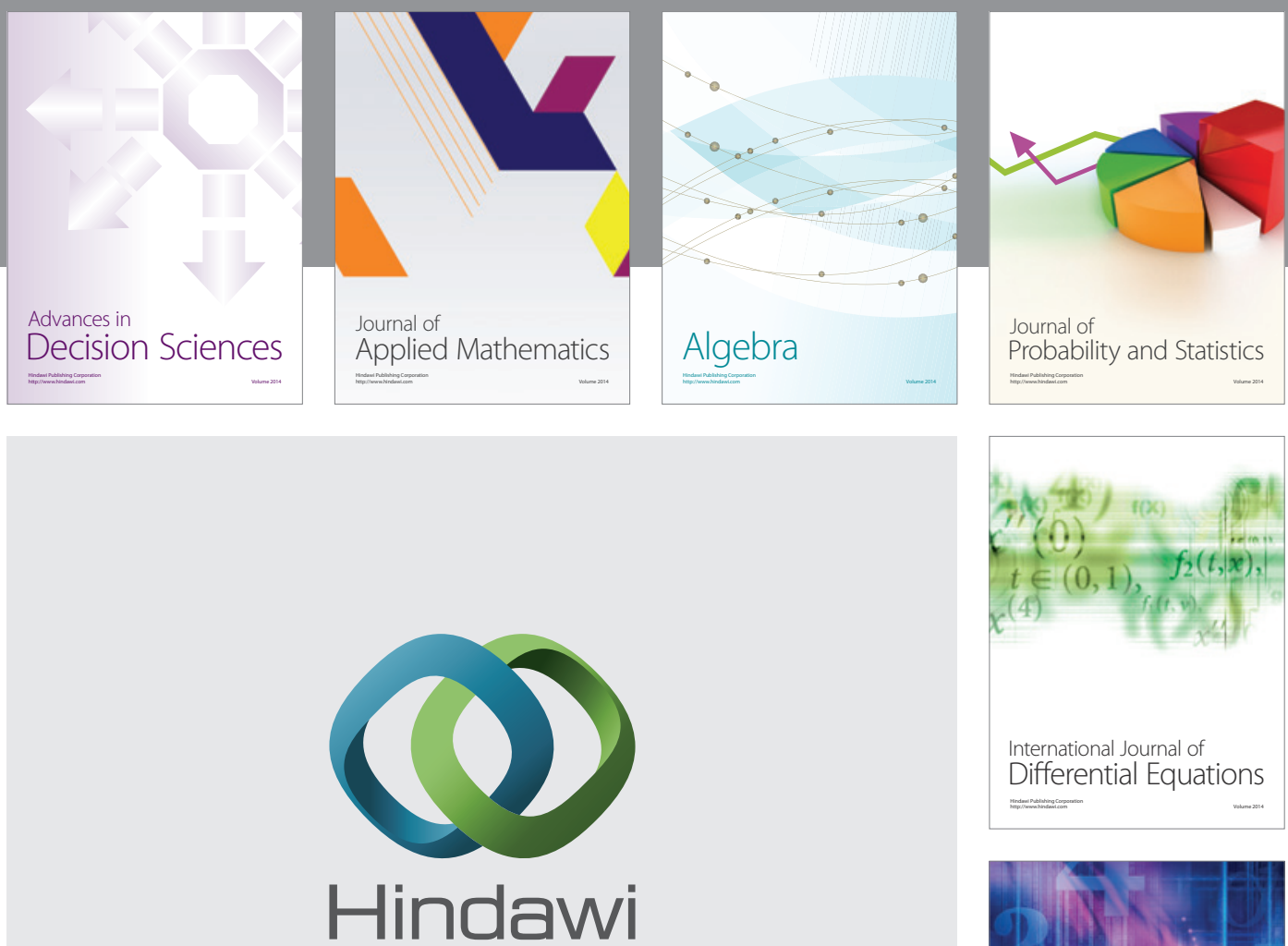

Submit your manuscripts at http://www.hindawi.com
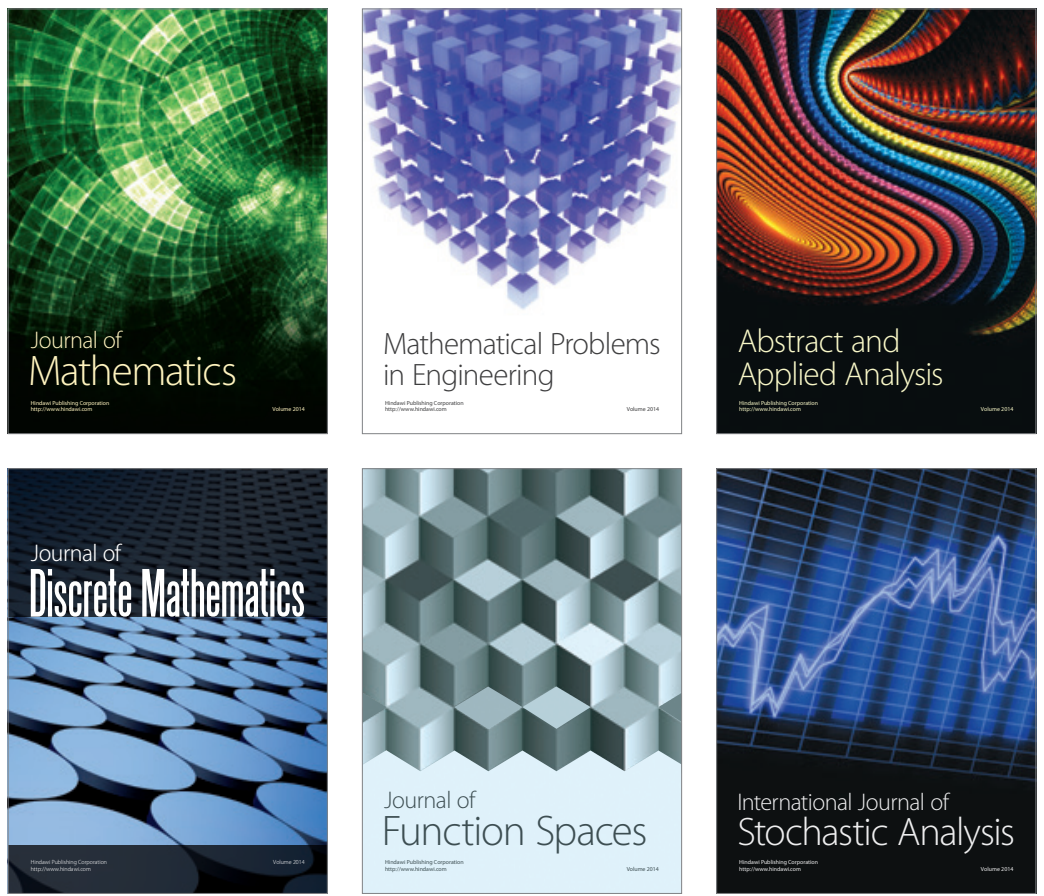

Journal of

Function Spaces

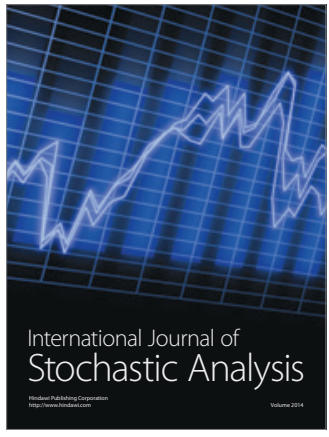

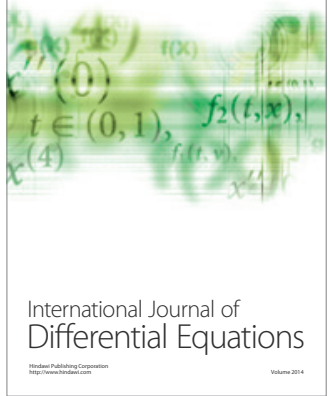
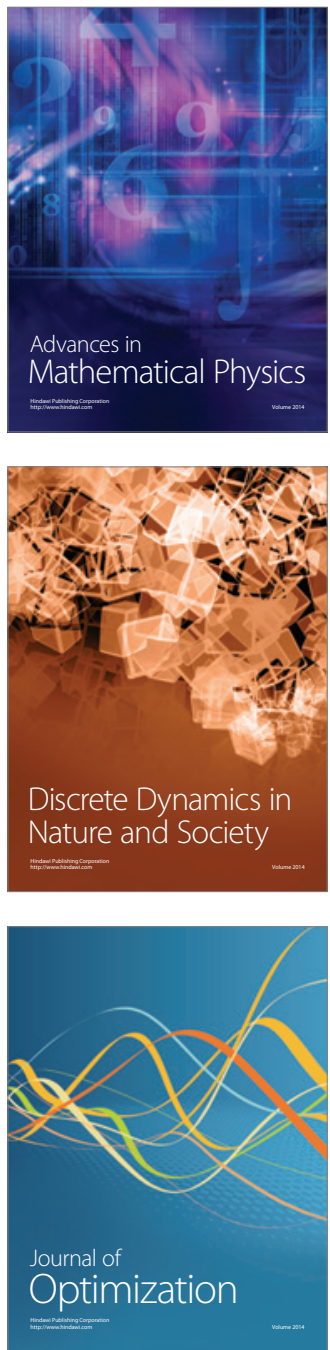